PROCEEDINGS OF THE

AMERICAN MATHEMATICAL SOCIETY

Volume 133, Number 8, Pages 2419-2428

S 0002-9939(05)07801-9

Article electronically published on February 25, 2005

\title{
A NOTE ON GABOR ORTHONORMAL BASES
}

\author{
YUN-ZHANG LI
}

(Communicated by Joseph A. Ball)

\begin{abstract}
The study of Gabor bases of the form $\left\{e^{-2 \pi i\langle\lambda, \cdot \cdot} g(\cdot-m): \lambda, m \in\right.$ $\left.\mathbb{Z}^{n}\right\}$ for $L^{2}\left(\mathbb{R}^{n}\right)$ has interested many mathematicians in recent years. Alex Losevich and Steen Pedersen in 1998, Jeffery C. Lagarias, James A. Reeds and Yang Wang in 2000 independently proved that, for any fixed positive integer $n,\left\{e^{-2 \pi i\langle\lambda, \cdot\rangle}: \lambda \in \Lambda\right\}$ is an orthonormal basis for $L^{2}\left([0,1]^{n}\right)$ if and only if $\left\{[0,1]^{n}+\lambda: \lambda \in \Lambda\right\}$ is a tiling of $\mathbb{R}^{n}$. Palle E. T. Jorgensen and Steen Pedersen in 1999 gave an explicit characterization of such $\Lambda$ for $n=1,2$, 3. Inspired by their work, this paper addresses Gabor orthonormal bases of the form $\left\{e^{-2 \pi i\langle\lambda, \cdot\rangle} g(\cdot-m): \lambda \in \Lambda, m \in \mathbb{Z}^{n}\right\}$ for $L^{2}\left(\mathbb{R}^{n}\right)$ and some other related problems, where $\Lambda$ is as above. For a fixed $n \in\{1,2,3\}$, the generating function $g$ of a Gabor orthonormal basis for $L^{2}\left(\mathbb{R}^{n}\right)$ corresponding to the above $\Lambda$ is characterized explicitly provided that $\operatorname{supp}(g)=\left[a_{1}, b_{1}\right] \times \cdots \times\left[a_{n}, b_{n}\right]$, which is new even if $\Lambda=\mathbb{Z}^{n}$; a Shannon type sampling theorem about such $\Lambda$ is derived when $n=2,3$; for an arbitrary positive integer $n$, an explicit expression of the $g$ with $\left\{e^{-2 \pi i\langle\lambda, \cdot\rangle} g(\cdot-m): \lambda, m \in \mathbb{Z}^{n}\right\}$ being an orthonormal basis for $L^{2}\left(\mathbb{R}^{n}\right)$ is obtained under the condition that $|\operatorname{supp}(g)|=1$.
\end{abstract}

\section{INTRODUCTION}

Let $n$ be a fixed positive integer. For $x \in \mathbb{R}^{n}$ and $1 \leq i \leq n, x_{i}$ denotes the $i$-th coordinate of $x$. For a Lebesgue measurable set $S$ in $\mathbb{R}^{n},|S|$ denotes its Lebesgue measure. For a Lebesgue measurable function $f$ on $\mathbb{R}^{n}, \operatorname{supp}(f)$ denotes the closure of the set $\left\{x \in \mathbb{R}^{n}: f(x) \neq 0\right\}$ in $\mathbb{R}^{n}$. The Fourier transform $\hat{f}$ of an $f \in L^{1}\left(\mathbb{R}^{n}\right)$ is defined by

$$
\hat{f}(\cdot)=\int_{\mathbb{R}^{n}} d x f(x) e^{-2 \pi i\langle x, \cdot\rangle} .
$$

Let $Q$ be a Lebesgue measurable set in $\mathbb{R}^{n}$ and $\Lambda$ a set in $\mathbb{R}^{n}$. $Q$ is called congruent to $[0,1]^{n}$ modulo $\mathbb{Z}^{n}$ if $|Q|=1$, and if to almost every $x \in[0,1]^{n}$ there corresponds a $k \in \mathbb{Z}^{n}$ such that $x+k \in Q$; the collection of sets $\{Q+\lambda: \lambda \in \Lambda\}$ is called a tiling of $\mathbb{R}^{n}$ if

$$
\bigcup_{\lambda \in \Lambda}(Q+\lambda)=\mathbb{R}^{n} \text { up to a set of measure } 0 \text { and }\left|\left(Q+\lambda_{1}\right) \cap\left(Q+\lambda_{2}\right)\right|=0
$$

for any two distinct $\lambda_{1}, \lambda_{2} \in \Lambda$.

Received by the editors December 3, 2003 and, in revised form, April 19, 2004.

2000 Mathematics Subject Classification. Primary 42C40.

Key words and phrases. Gabor orthonormal basis, tiling.

This research was supported by the National Natural Science Foundation of China, and the Natural Science Foundation of Beijing.

(c)2005 American Mathematical Society Reverts to public domain 28 years from publication 2419 
Alex Losevich and Steen Pedersen in 1998, Jeffery C. Lagarias, James A. Reeds and Yang Wang in 2000 independently obtained that

Proposition 1.1 ([1. Theorem 1.1], [18 Theorem 1.1]). Let $n$ be a fixed positive integer, and $\Lambda$ a set in $\mathbb{R}^{n}$. Then $\left\{e^{-2 \pi i\langle\lambda, \cdot\rangle}: \lambda \in \Lambda\right\}$ is an orthonormal basis for $L^{2}\left([0,1]^{n}\right)$ if and only if $\left\{[0,1]^{n}+\lambda: \lambda \in \Lambda\right\}$ is a tiling of $\mathbb{R}^{n}$.

This proposition is significant because it reduces the construction of exponential orthonormal bases for $L^{2}\left([0,1]^{n}\right)$ to that of tilings of $\mathbb{R}^{n}$. In 1930 , Keller proved in [3] that

Proposition 1.2. Let $n$ be a fixed positive integer, and $\left\{[0,1]^{n}+\lambda: \lambda \in \Lambda\right\} a$ tiling of $\mathbb{R}^{n}$. Then each pair of distinct $\lambda, \lambda^{\prime} \in \Lambda$ has

$$
\lambda_{i}-\lambda_{i}^{\prime} \in \mathbb{Z} \backslash 0 \quad \text { for some } i, 1 \leq i \leq n .
$$

Put $n=1$. Then Proposition 1.1 and Proposition 1.2 yield that

Proposition 1.3. $\left\{e^{-2 \pi i\langle\lambda, \cdot\rangle}: \lambda \in \Lambda\right\}$ is an orthonormal basis for $L^{2}([0,1])$ if and only if $\Lambda=\mathbb{Z}+x_{0}$ for some $x_{0} \in \mathbb{R}$.

Proposition 1.3 shows that exponential orthonormal bases for $L^{2}([0,1])$ are essentially only of the form $\left\{e^{-2 \pi i\langle\lambda, \cdot\rangle}: \lambda \in \mathbb{Z}\right\}$ up to a factor of a unimodular Lebesgue measurable function, which was also given without detailed proof in [2]. But they are complicated in high dimensions since there are many exotic cube tilings at that time (4]). By [2, Theorem 5 and Theorem 6] and a careful investigation of their proofs, we have

Proposition 1.4. $\left\{e^{-2 \pi i\langle\lambda, \cdot\rangle}: \lambda \in \Lambda\right\}$ is an orthonormal basis for $L^{2}\left([0,1]^{2}\right)$ if and only if

$\Lambda=\left\{\left(\alpha+k_{1}, \beta\left(k_{1}\right)+k_{2}\right)^{T}: k_{1}, k_{2} \in \mathbb{Z}\right\}$ or $\left\{\left(\beta\left(k_{2}\right)+k_{1}, \alpha+k_{2}\right)^{T}: k_{1}, k_{2} \in \mathbb{Z}\right\}$

for some $\alpha \in \mathbb{R}$ and some sequence $\beta$ of real numbers on $\mathbb{Z}$.

Proposition 1.5. $\left\{e^{-2 \pi i\langle\lambda, \cdot\rangle}: \lambda \in \Lambda\right\}$ is an orthonormal basis for $L^{2}\left([0,1]^{3}\right)$ if and only if, up to a possible translation by a single vector and a possible permutation of coordinates,

$$
\begin{aligned}
\Lambda & =\left\{\left(a, \alpha_{0}(a)+k_{2}, \alpha_{1}\left(a, k_{2}\right)+k_{3}\right)^{T}: a \in \mathbb{A}, k_{2}, k_{3} \in \mathbb{Z}\right\} \\
& +\cup\left\{\left(b, \beta_{1}\left(b, k_{3}\right)+k_{2}, \beta_{0}(b)+k_{3}\right)^{T}: b \in \mathbb{B}, k_{2}, k_{3} \in \mathbb{Z}\right\},
\end{aligned}
$$

where $\mathbb{A}$ and $\mathbb{B}$ are such that $\mathbb{A} \cap \mathbb{B}=\emptyset$ and $\mathbb{A} \cup \mathbb{B}=\mathbb{Z}$ (one possibly empty), $\alpha_{0}$, $\beta_{0}, \alpha_{1}$ and $\beta_{1}$ are real functions defined on $\mathbb{A}, \mathbb{B}, \mathbb{A} \times \mathbb{Z}$ and $\mathbb{B} \times \mathbb{Z}$, respectively.

Definition 1.1. Let $g \in L^{2}\left(\mathbb{R}^{n}\right)$ and $\Lambda \subset \mathbb{R}^{n} .\left\{e^{-2 \pi i\langle\lambda, \cdot\rangle} g(\cdot-m): \lambda \in \Lambda, m \in\right.$ $\left.\mathbb{Z}^{n}\right\}$ is called a Gabor orthonormal basis for $L^{2}\left(\mathbb{R}^{n}\right)$ if it is an orthonormal basis for $L^{2}\left(\mathbb{R}^{n}\right)$.

In this paper, the generating function $g$ of a Gabor orthonormal basis for $L^{2}\left(\mathbb{R}^{n}\right)$ corresponding to an orthonormal basis $\left\{e^{-2 \pi i\langle\lambda, \cdot\rangle}: \lambda \in \Lambda\right\}$ for $L^{2}\left([0,1]^{n}\right)(n=1$, $2,3)$ is characterized explicitly provided that $\operatorname{supp}(g)=\left[a_{1}, b_{1}\right] \times \cdots \times\left[a_{n}, b_{n}\right]$, which is new even if $\Lambda=\mathbb{Z}^{n}$; a corresponding Shannon type sampling theorem about such $\Lambda$ is derived; for an arbitrary positive integer $n$, an explicit expression of the $g$ with $\left\{e^{-2 \pi i\langle\lambda, \cdot\rangle} g(\cdot-m): \lambda, m \in \mathbb{Z}^{n}\right\}$ being a Gabor orthonormal basis for $L^{2}\left(\mathbb{R}^{n}\right)$ is obtained under the condition that $|\operatorname{supp}(g)|=1$. The other related results of Gabor bases can be found in [5]-[15], [19] and [20]. Our main results can be stated as follows. 
Theorem 1.1. Let $n \in\{1,2,3\}$, and let $\left\{e^{-2 \pi i\langle\lambda, \cdot\rangle}: \lambda \in \Lambda\right\}$ be an orthonormal basis for $L^{2}\left([0,1]^{n}\right)$. Then, for $g \in L^{2}\left(\mathbb{R}^{n}\right)$ satisfying $\operatorname{supp}(g)=\left[a_{1}, b_{1}\right] \times \cdots \times$ $\left[a_{n}, b_{n}\right],\left\{e^{-2 \pi i\langle\lambda \cdot \cdot\rangle} g(\cdot-m): \lambda \in \Lambda, m \in \mathbb{Z}^{n}\right\}$ is an orthonormal basis for $L^{2}\left(\mathbb{R}^{n}\right)$ if and only if $g(\cdot)=h(\cdot) \chi_{[0,1]^{n}+\tilde{x}}(\cdot)$ a.e. on $\mathbb{R}^{n}$ for some $\tilde{x} \in \mathbb{R}^{n}$ and some unimodular Lebesgue measurable function $h$ on $\mathbb{R}^{n}$.

Theorem 1.2. (1) Let $\alpha \in \mathbb{R}$, let $\beta$ be a sequence of real numbers defined on $\mathbb{Z}$, and let $\lambda_{k}=\left(\alpha+k_{1}, \beta\left(k_{1}\right)+k_{2}\right)^{T}$ for $k \in \mathbb{Z}^{2}$. Then in the sense of pointwise convergence,

$$
f(x)=\sum_{k \in \mathbb{Z}^{2}} f\left(\lambda_{k}\right) \frac{\sin \pi\left(x_{1}-k_{1}-\alpha\right) \sin \pi\left(x_{2}-k_{2}-\beta\left(k_{1}\right)\right)}{\pi^{2}\left(x_{1}-k_{1}-\alpha\right)\left(x_{2}-k_{2}-\beta\left(k_{1}\right)\right)}
$$

on $\mathbb{R}^{2}$ for any continuous function $f$ satisfying $f \in L^{2}\left(\mathbb{R}^{2}\right)$ and $\operatorname{supp}(\hat{f}) \subset\left[-\frac{1}{2}, \frac{1}{2}\right]^{2}$.

(2) Let $\mathbb{A}$ and $\mathbb{B}$ satisfy $\mathbb{A} \cap \mathbb{B}=\emptyset$ and $\mathbb{A} \cup \mathbb{B}=\mathbb{Z}$ (one possibly empty), let $\alpha_{0}, \beta_{0}, \alpha_{1}$ and $\beta_{1}$ be respectively real functions defined on $\mathbb{A}, \mathbb{B}, \mathbb{A} \times \mathbb{Z}$ and $\mathbb{B} \times \mathbb{Z}$, let $\lambda_{k}=\left(k_{1}, \alpha_{0}\left(k_{1}\right)+k_{2}, \alpha_{1}\left(k_{1}, k_{2}\right)+k_{3}\right)^{T}$ for $k \in \mathbb{A} \times \mathbb{Z}^{2}$, and let $\mu_{k}=$ $\left(k_{1}, \beta_{1}\left(k_{1}, k_{3}\right)+k_{2}, \beta_{0}\left(k_{1}\right)++k_{3}\right)^{T}$ for $k \in \mathbb{B} \times \mathbb{Z}^{2}$. Then in the sense of pointwise convergence,

$$
\begin{aligned}
& f(x)= \\
& \sum_{k \in \mathbb{A} \times \mathbb{Z}^{2}} f\left(\lambda_{k}\right) \frac{\sin \pi\left(x_{1}-k_{1}\right) \sin \pi\left(x_{2}-k_{2}-\alpha_{0}\left(k_{1}\right)\right) \sin \pi\left(x_{3}-k_{3}-\alpha_{1}\left(k_{1}, k_{2}\right)\right)}{\pi^{3}\left(x_{1}-k_{1}\right)\left(x_{2}-k_{2}-\alpha_{0}\left(k_{1}\right)\right)\left(x_{3}-k_{3}-\alpha_{1}\left(k_{1}, k_{2}\right)\right)} \\
& +\sum_{k \in \mathbb{B} \times \mathbb{Z}^{2}} f\left(\mu_{k}\right) \frac{\sin \pi\left(x_{1}-k_{1}\right) \sin \pi\left(x_{2}-k_{2}-\beta_{1}\left(k_{1}, k_{3}\right)\right) \sin \pi\left(x_{3}-k_{3}-\beta_{0}\left(k_{1}\right)\right)}{\pi^{3}\left(x_{1}-k_{1}\right)\left(x_{2}-k_{2}-\beta_{1}\left(k_{1}, k_{3}\right)\right)\left(x_{3}-k_{3}-\beta_{0}\left(k_{1}\right)\right)}
\end{aligned}
$$

on $\mathbb{R}^{3}$ for any continuous function $f$ satisfying $f \in L^{2}\left(\mathbb{R}^{3}\right)$ and $\operatorname{supp}(\hat{f}) \subset\left[-\frac{1}{2}, \frac{1}{2}\right]^{3}$.

Remark 1.1. The one-dimensional Shannon Theorem is well known, which can also be found in [5]. This theorem can be regarded as the two- and three-dimensional Shannon Theorem. This theorem shows that $\lambda_{k}$ in (1), $\lambda_{k}$ and $\mu_{k}$ in (2) are both a set of sampling points and a set of interpolation points for continuous band-limited functions with $\operatorname{supp}(\hat{f})=\left[-\frac{1}{2}, \frac{1}{2}\right]^{2}$ and $\left[-\frac{1}{2}, \frac{1}{2}\right]^{3}$, respectively.

Theorem 1.3. Let $n$ be a fixed positive integer, $g \in L^{2}\left(\mathbb{R}^{n}\right)$, and $|\operatorname{supp}(g)|=1$. Then $\left\{e^{-2 \pi i\langle\lambda, \cdot\rangle} g(\cdot-m): \lambda, m \in \mathbb{Z}^{n}\right\}$ is an orthonormal basis for $L^{2}\left(\mathbb{R}^{n}\right)$ if and only if $g(\cdot)=h(\cdot) \chi_{Q}(\cdot)$ a.e. on $\mathbb{R}^{n}$ for some $Q$ congruent to $[0,1]^{n}$ modulo $\mathbb{Z}^{n}$ and some unimodular Lebesgue measurable function $h$ on $\mathbb{R}^{n}$.

Remark 1.2. Applying a similar procedure as in Step 1 of "Proof of Theorem 1.1" and Lemma 2.2 below, we immediately have the following corollary:

Corollary 1.1. Let $n$ be an arbitrary positive integer, and let $g$ be a function in $L^{2}\left(\mathbb{R}^{n}\right)$ satisfying $\operatorname{supp}(g)=\left[a_{1}, b_{1}\right] \times \cdots \times\left[a_{n}, b_{n}\right]$. Then $\left\{e^{-2 \pi i\langle\lambda, \cdot\rangle} g(\cdot-m)\right.$ : $\left.\lambda, m \in \mathbb{Z}^{n}\right\}$ is an orthonormal basis for $L^{2}\left(\mathbb{R}^{n}\right)$ if and only if $g(\cdot)=h(\cdot) \chi_{[0,1]^{n}+\tilde{x}}(\cdot)$ for some $\tilde{x} \in \mathbb{R}^{n}$ and some unimodular Lebesgue measurable function $h$ on $\mathbb{R}^{n}$.

From Theorem 1.1, we know that the corollary is true when $\lambda$ ranges over an arbitrary index set $\Lambda$ with $\left\{e^{-2 \pi i\langle\lambda, \cdot\rangle}: \lambda \in \Lambda\right\}$ being an orthonormal basis for $L^{2}\left([0,1]^{n}\right)$ if $n \leq 3$. But it is unresolved whether it is true when $\lambda$ ranges over an arbitrary index set $\Lambda$ with $\left\{e^{-2 \pi\langle\lambda, \cdot\rangle}: \lambda \in \Lambda\right\}$ being an orthonormal basis for $L^{2}\left([0,1]^{n}\right)$ if $n>3$. 
This paper is organized as follows. In Section 2, some auxiliary lemmas are obtained; in Section 3, the proofs of the theorems are given.

\section{Some AUXILIARY LEMMAS}

Lemma 2.1. Let $n$ be a fixed positive integer, and $Q$ a Lebesgue measurable set in $\mathbb{R}^{n}$ such that $\bigcup_{k \in \mathbb{Z}^{n}}(Q+k)=\mathbb{R}^{n}$ up to a set of measure 0 . Then $|Q| \geq 1$.

Proof. It is obvious that

$$
\begin{aligned}
|Q| & =\int_{\mathbb{R}^{n}} d x \chi_{Q}(x) \sum_{k \in \mathbb{Z}^{n}} \chi_{[0,1]^{n}}(x+k) \\
& =\sum_{k \in \mathbb{Z}^{n}} \int_{\mathbb{R}^{n}} d x \chi_{Q}(x) \chi_{[0,1]^{n}}(x+k) \\
& =\sum_{k \in \mathbb{Z}^{n}} \int_{\mathbb{R}^{n}} d x \chi_{Q}(x-k) \chi_{[0,1]^{n}}(x) \\
& =\int_{\mathbb{R}^{n}} d x \chi_{[0,1]^{n}}(x) \sum_{k \in \mathbb{Z}^{n}} \chi_{Q}(x-k) .
\end{aligned}
$$

Noting that $\sum_{k \in \mathbb{Z}^{n}} \chi_{Q}(\cdot-k) \geq 1$ a.e. on $\mathbb{R}^{n}$ since $\bigcup_{k \in \mathbb{Z}^{n}}(Q+k)=\mathbb{R}^{n}$ up to a set of measure 0 , we have $|Q| \geq 1$. The proof is completed.

Lemma 2.2. Let $n$ be a fixed positive integer, $a_{i}, b_{i} \in \mathbb{R}, a_{i}<b_{i}$ for $i=1,2, \cdots, n$, and $Q=\left[a_{1}, b_{1}\right] \times \cdots \times\left[a_{n}, b_{n}\right]$. Then $\left\{Q+k: k \in \mathbb{Z}^{n}\right\}$ is a tiling of $\mathbb{R}^{n}$ if and only if $Q=[0,1]^{n}+\tilde{x}$ for some $\tilde{x} \in \mathbb{R}^{n}$ up to a set of measure 0 .

Proof. Sufficiency is obvious. Necessity is reduced to $b_{1}-a_{1}=b_{2}-a_{2}=\cdots=$ $b_{n}-a_{n}=1$. We show it by contradiction. Without loss of generality, we assume that $b_{1}-a_{1} \neq 1$. Then $\left\{x \in \mathbb{R}^{n}: b_{1}<x_{1}<a_{1}+1\right\} \subset \mathbb{R}^{n} \backslash \bigcup_{k \in \mathbb{Z}^{n}}(Q+k)$ if $b_{1}-a_{1}<1$, and $\left|Q \cap\left(Q+(1,0, \cdots, 0)^{T}\right)\right|>0$ if $b_{1}-a_{1}>1$. This contradicts the fact that $\left\{Q+k: k \in \mathbb{Z}^{n}\right\}$ is a tiling of $\mathbb{R}^{n}$. The proof is completed.

Lemma 2.3. Let $n$ be a fixed positive integer, and $Q$ a Lebesgue measurable set in $\mathbb{R}^{n}$. Then $\left\{Q+k: k \in \mathbb{Z}^{n}\right\}$ is a tiling of $\mathbb{R}^{n}$ if and only if $Q$ is congruent to $[0,1]^{n}$ modulo $\mathbb{Z}^{n}$.

Proof. Necessity. We have $|Q|=1$ by [17, Lemma 1]. This together with the definition of tiling finishes the proof of the necessity.

Sufficiency. By [17, Lemma 1], it suffices to show that $\bigcup_{k \in \mathbb{Z}^{n}}(Q+k)=\mathbb{R}^{n}$ up to a set of measure 0 . To almost every $x \in \mathbb{R}^{n}$ there corresponds an $l \in \mathbb{Z}^{n}$ such that $x-l \in[0,1]^{n}$, and thus $x-l+m \in Q$ for some $m \in \mathbb{Z}^{n}$ since $Q$ is congruent to $[0,1]^{n}$ modulo $\mathbb{Z}^{n}$, which finishes the proof of the sufficiency.

Lemma 2.4. Let $n \in\{1,2,3\}$, let $\left\{e^{-2 \pi i\langle\lambda, \cdot\rangle}: \lambda \in \Lambda\right\}$ be an orthonormal basis for $L^{2}\left([0,1]^{n}\right)$, let $f \in L^{1}\left([0,1]^{n}\right.$, and let $\int_{[0,1]^{n}} d x f(x) e^{2 \pi i\langle\lambda, x\rangle}=0$ for $\lambda \in \Lambda$. Then $f(\cdot)=0$ a.e. on $[0,1]^{n}$.

Proof. The lemma is trivial for $n=1$ by [16, Theorem 2.4.1]. In what follows, we only prove the lemma for $n=2$, and the case of $n=3$ can be proved analogously.

By Proposition 1.4, without loss of generality, we assume that

$$
\Lambda=\left\{\left(\alpha+k_{1}, \beta\left(k_{1}\right)+k_{2}\right)^{T}: k_{1}, k_{2} \in \mathbb{Z}\right\}
$$


for some $\alpha \in \mathbb{R}$ and some sequence $\beta$ of real numbers on $\mathbb{Z}$. Then

$$
\begin{aligned}
& \int_{[0,1]} d x_{2} e^{2 \pi i k_{2} x_{2}} e^{2 \pi i \beta\left(k_{1}\right) x_{2}} \int_{[0,1]} d x_{1} e^{2 \pi i\left(k_{1}+\alpha\right) x_{1}} f\left(x_{1}, x_{2}\right) \\
& =\int_{[0,1]^{2}} d x_{1} d x_{2} f\left(x_{1}, x_{2}\right) e^{2 \pi i\left[\left(k_{1}+\alpha\right) x_{1}+\left(\beta\left(k_{1}\right)+k_{2}\right) x_{2}\right]} \\
& =0
\end{aligned}
$$

for $k_{2} \in \mathbb{Z}$ and any fixed $k_{1} \in \mathbb{Z}$. It follows from [16, Theorem 2.4.1] that

$$
\int_{[0,1]} d x_{1} e^{2 \pi i\left(k_{1}+\alpha\right) x_{1}} f\left(x_{1}, x_{2}\right)=0
$$

for almost all $x_{2} \in[0,1]$ and any fixed $k_{1} \in \mathbb{Z}$. Applying [16. Theorem 2.4.1] again gives $e^{2 \pi i \alpha x_{1}} f\left(x_{1}, x_{2}\right)=0$ a.e. on $[0,1]^{2}$, which implies $f(\cdot)=0$ a.e. on $[0,1]^{2}$. The proof is completed.

Remark 2.1. It is unresolved whether the conclusion of this lemma holds when $n>3$ since there are many exotic index sets $\Lambda$ at this time (4 4 ).

Lemma 2.5. Let $n$ be a fixed positive integer, $g \in L^{2}\left(\mathbb{R}^{n}\right)$, and $\left\{e^{-2 \pi i\langle\lambda, \cdot\rangle} g(\cdot-m)\right.$ : $\left.\lambda \in \Lambda, m \in \mathbb{Z}^{n}\right\}$ complete in $L^{2}\left(\mathbb{R}^{n}\right)$. Then $\bigcup_{m \in \mathbb{Z}^{n}}[\operatorname{supp}(g)+m]=\mathbb{R}^{n}$ up to a set of measure 0 .

Proof. By contradiction. Otherwise, there exists a set $S$ of positive finite measure such that $|S \cap[\operatorname{supp}(g)+m]|=0$ for $m \in \mathbb{Z}^{n}$. It follows that

$$
\int_{\mathbb{R}^{n}} d x \chi_{S}(x) e^{2 \pi i\langle\lambda, x\rangle} \overline{g(x-m)}=0 \text { for } m \in \mathbb{Z}^{n},
$$

which is a contradiction. The proof is completed.

Lemma 2.6. Let $n$ be a positive integer, and $Q$ congruent to $[0,1]^{n}$ modulo $\mathbb{Z}^{n}$. Then $\left\{e^{-2 \pi i\langle\lambda, \cdot\rangle}: \lambda \in \mathbb{Z}^{n}\right\}$ is an orthonormal basis for $L^{2}(Q)$.

Proof. Define $I_{Q}=\left\{\lambda \in \mathbb{Z}^{n}: \lambda+x \in Q\right.$ for some $\left.x \in[0,1]^{n}\right\}$. Then $I_{Q}$ is an at most countable set. Write $I_{Q}=\left\{\lambda_{1}, \lambda_{2}, \cdots, \lambda_{j}, \cdots\right\}$. Define $S_{j}=\left\{x \in[0,1]^{n}\right.$ : $\left.\lambda_{j}+x \in[0,1]^{n}\right\}$, and $Q_{j}=S_{j}+\lambda_{j}$ for $\lambda_{j} \in I_{Q}$. Then, for $\lambda \in \mathbb{Z}^{n}$,

$$
\begin{aligned}
& \int_{Q} d x e^{-2 \pi i\langle\lambda, x\rangle} \\
& =\int_{\cup_{j} Q_{j}} d x e^{-2 \pi i\langle\lambda, x\rangle} \\
& =\sum_{j} \int_{S_{j}+\lambda_{j}} d x e^{-2 \pi i\langle\lambda, x\rangle} \\
& =\sum_{j} \int_{S_{j}} d x e^{-2 \pi i\langle\lambda, x\rangle} \\
& =\int_{[0,1]^{n}} d x e^{-2 \pi i\langle\lambda, x\rangle} \\
& =\delta_{\lambda, 0} .
\end{aligned}
$$

Note that $|Q|=1$. It follows that $\left\{e^{-2 \pi i\langle\lambda, \cdot\rangle}: \lambda \in \mathbb{Z}^{n}\right\}$ is an orthonormal system for $L^{2}(Q)$. In what follows, we show its completeness. 
Suppose $\int_{Q} d x f(x) e^{2 \pi i\langle\lambda, x\rangle}=0$ for all $\lambda \in \mathbb{Z}^{n}$ and some $f \in L^{2}(Q)$. Applying Lebesgue's Dominated Convergence Theorem, we have

$$
\begin{aligned}
& \int_{Q} d x f(x) e^{2 \pi i\langle\lambda, x\rangle} \\
& =\sum_{j} \int_{S_{j}+\lambda_{j}} d x f(x) e^{2 \pi i\langle\lambda, x\rangle} \\
& =\sum_{j} \int_{[0,1]^{n}} d x f\left(x+\lambda_{j}\right) \chi_{S_{j}}(x) e^{2 \pi i\langle\lambda, x\rangle} \\
& =\int_{[0,1]^{n}} d x\left(\sum_{j} f\left(x+\lambda_{j}\right) \chi_{S_{j}}(x)\right) e^{2 \pi i\langle\lambda, x\rangle} .
\end{aligned}
$$

So $\int_{[0,1]^{n}} d x\left(\sum_{j} f\left(x+\lambda_{j}\right) \chi_{S_{j}}(x)\right) e^{2 \pi i\langle\lambda, x\rangle}=0$ for $\lambda \in \mathbb{Z}^{n}$. Applying [16, Theorem 2.4.1] it follows that $\sum_{j} f\left(\cdot+\lambda_{j}\right) \chi_{S_{j}}(\cdot)=0$ a.e. on $[0,1]^{n}$, which implies $f(\cdot)=0$ a.e. on $Q$ by the definition of $S_{j}$ and $Q_{j}$. The completeness is proved.

\section{Proof OF THE THEOREMS}

Proof of Theorem 1.1. We only prove the theorem for $n=2$, since the other two cases can be proved similarly. First we divide the proof into two steps to prove the necessity.

Step 1. $\operatorname{supp}(g)=[0,1]^{2}+\tilde{x}$ for some $\tilde{x} \in \mathbb{R}^{n}$.

It suffices to show that $\left\{[0,1]^{2}+m: m \in \mathbb{Z}^{2}\right\}$ is a tiling of $\mathbb{R}^{2}$ by Lemma 2.2 . Applying Lemma 2.5 leads to $\bigcup_{m \in \mathbb{Z}^{2}}[\operatorname{supp}(g)+m]=\mathbb{R}^{2}$ up to a set of measure 0 , and thus $|\operatorname{supp}(g)| \geq 1$ by Lemma 2.1. So, by [16] Lemma 1], it is enough to show that $|\operatorname{supp}(g)|=1$. Now we show it by contradiction. $\operatorname{Suppose}|\operatorname{supp}(g)|>1$. Then there exists some $0 \neq l \in \mathbb{Z}^{2}$ such that $0<b_{i}-a_{i}-l_{i} \leq 1$ for $b_{i}-a_{i}>1$, and $l_{i}=0$ for $b_{i}-a_{i} \leq 1$. It follows that

$$
|\operatorname{supp}(g) \cap[\operatorname{supp}(g)-l]|>0 .
$$

By Proposition 1.4, without loss of generality, we assume that

$$
\Lambda=\left\{\left(\alpha+k_{1}, \beta\left(k_{1}\right)+k_{2}\right)^{T}: k_{1}, k_{2} \in \mathbb{Z}\right\}
$$

for some $\alpha \in \mathbb{R}$ and some sequence $\beta$ of real numbers on $\mathbb{Z}$. Write $G(\cdot)=\overline{g(\cdot)} g(\cdot+l)$. By the orthogonality of $\left\{e^{-2 \pi i\langle\lambda, \cdot\rangle} g(\cdot-m): \lambda \in \Lambda, m \in \mathbb{Z}^{2}\right\}$, for $k \in \mathbb{Z}^{2}$,

$$
\begin{aligned}
& \int_{[0,1]^{2}} e^{-2 \pi i\left\langle\left(\begin{array}{c}
k_{1}+\beta \\
k_{2}+\beta\left(k_{1}\right)-\beta(0)
\end{array}\right), x\right\rangle} G(x) \\
& =\int_{[0,1]^{2}} e^{-2 \pi i\left\langle\left(\begin{array}{c}
k_{1}+\alpha \\
k_{2}+\beta\left(k_{1}\right)
\end{array}\right), x\right\rangle} g(x+l) \overline{e^{-2 \pi i\left\langle\left(\begin{array}{c}
\alpha \\
\beta(0)
\end{array}\right), x\right\rangle} g(x)} \\
& =0 .
\end{aligned}
$$

By Proposition 1.4, $\left\{e^{-2 \pi i\left\langle\left(\begin{array}{c}k_{1}+\beta\left(k_{1}\right)-\beta(0) \\ k_{2}\end{array}\right), \cdot\right\rangle}: k_{1}, k_{2} \in \mathbb{Z}\right\}$ is an orthonormal basis for $L^{2}\left([0,1]^{2}\right)$. Note that $\operatorname{supp}(G) \subset[0,1]^{2}$. From this, together with Lemma 2.4, it follows that $G(\cdot)=0$ a.e. on $\mathbb{R}^{2}$, which contradicts (3.1).

Step 2. $g(\cdot)=h(\cdot) \chi_{[0,1]^{n}+\tilde{x}}(\cdot)$ for some unimodular Lebesgue measurable function $h$ on $\mathbb{R}^{2}$. 
From the orthogonality of $\left\{e^{-2 \pi i\langle\lambda, \cdot\rangle} g(\cdot-m): \lambda \in \Lambda, m \in \mathbb{Z}^{2}\right\}$ and Step 1, it follows that $\int_{[0,1]^{2}+\tilde{x}}|g(x)|^{2}=1$,

$$
\begin{aligned}
& \int_{[0,1]^{2}+\tilde{x}} e^{-2 \pi i\left\langle\left(\begin{array}{c}
k_{k_{2}}+\beta\left(k_{1}\right)-\beta(0) \\
k^{2}
\end{array}\right), x\right\rangle}|g(x)|^{2} \\
& =\int_{\mathbb{R}^{2}} \overline{e^{-2 \pi i\left\langle\left(\begin{array}{c}
k_{1}+\alpha \\
k_{2}+\beta\left(k_{1}\right)
\end{array}\right), x\right\rangle} g(x)} e^{-2 \pi i\left\langle\left(\begin{array}{c}
\alpha \\
\beta(0)
\end{array}\right), x\right\rangle} g(x) \\
& =0
\end{aligned}
$$

for $0 \neq k \in \mathbb{Z}^{2}$, and thus $\int_{[0,1]^{2}+\tilde{x}} e^{-2 \pi i\left\langle\left(\begin{array}{c}k_{1} \\ k_{2}+\beta\left(k_{1}\right)-\beta(0)\end{array}\right), x\right\rangle}\left(|g(x)|^{2}-1\right)=0$ for $k \in \mathbb{Z}^{2}$. Note that $\left.\left.\left\{e_{k}(\cdot)=e^{-2 \pi i\left\langle\left(k_{2}+\beta\left(k_{1}\right)-\beta(0)\right.\right.}\right), \cdot\right\rangle: k_{1}, k_{2} \in \mathbb{Z}\right\}$ is an orthonormal basis for $L^{2}\left([0,1]^{2}\right)$ as in Step 1. It is also an orthonormal basis for $L^{2}\left([0,1]^{2}+\tilde{x}\right)$. So $|g(\cdot)|=1$ a.e. on $[0,1]^{2}+\tilde{x}$, which together with Step 1 leads to $|g(\cdot)|=$ $\chi_{[0,1]^{2}+\tilde{x}}(\cdot)$ a.e. on $\mathbb{R}^{2}$. Define

$$
h(x)= \begin{cases}\frac{g(x)}{|g(x)|}, & \text { if } g(x) \neq 0 \\ 1, & \text { if } g(x)=0\end{cases}
$$

Then $g(\cdot)=h(\cdot) \chi_{[0,1]^{n}+\tilde{x}}(\cdot)$, and $h$ is as desired.

Now we prove the sufficiency. Note that $\left\{e^{-2 \pi i\langle\lambda, \cdot\rangle}: \lambda \in \Lambda\right\}$ is also an orthonormal basis for $L^{2}\left([0,1]^{2}+\tilde{x}\right)$. It is easy to check that

$$
\left\{e^{-2 \pi i\langle\lambda, \cdot\rangle} g(\cdot-m): \lambda \in \Lambda, m \in \mathbb{Z}^{2}\right\}
$$

is an orthonormal system in $L^{2}\left(\mathbb{R}^{2}\right)$. So it suffices to show its completeness in $L^{2}\left(\mathbb{R}^{2}\right)$. Suppose there is an $f \in L^{2}\left(\mathbb{R}^{2}\right)$ such that $\int_{\mathbb{R}^{2}} f(x) e^{2 \pi i\langle\lambda, x\rangle} \overline{g(x-m)}=0$ for $\lambda \in \Lambda$ and $m \in \mathbb{Z}^{2}$. Then, for an arbitrarily fixed $m \in \mathbb{Z}^{2}$,

$$
\int_{[0,1]^{2}+\tilde{x}} f(x+m) \overline{h(x)} e^{2 \pi i\langle\lambda, x+m\rangle}=0
$$

for $\lambda \in \Lambda$. Note that $\left\{e^{-2 \pi i\langle\lambda, \cdot+m\rangle}: \lambda \in \Lambda\right\}$ is an orthonormal basis for $L^{2}\left([0,1]^{2}+\right.$ $\tilde{x})$. It follows that $f(\cdot+m) \overline{h(\cdot)}=0$, and thus $f(\cdot+m)=0$ a.e. on $[0,1]^{2}+\tilde{x}$ for $m \in \mathbb{Z}^{2}$, which implies $f(\cdot)=0$ a.e. on $\mathbb{R}^{2}$. The completeness is proved.

Proof of Theorem 1.2. We only prove (1), and (2) can be proved similarly. Suppose $f$ is a continuous function satisfying $f \in L^{2}\left(\mathbb{R}^{2}\right)$ and $\operatorname{supp}(\hat{f}) \subset\left[-\frac{1}{2}, \frac{1}{2}\right]^{2}$. We claim that

$$
f(\cdot)=\int_{\mathbb{R}^{2}} d \xi e^{2 \pi i\langle\cdot, \xi\rangle} \hat{f}(\xi) \text { on } \mathbb{R}^{2} \text { in the pointwise sense. }
$$

Indeed, the equation holds a.e. on $\mathbb{R}^{2}$ since $f \in L^{2}\left(\mathbb{R}^{2}\right)$. Note that the right side in the equation is an entire function on $\mathbb{R}^{2}$ since $\operatorname{supp}(\hat{f}) \subset\left[-\frac{1}{2}, \frac{1}{2}\right]^{2}$, and that $f$ is continuous on $\mathbb{R}^{2}$. Equation (3.2) follows.

By Theorem 1.1, $\left\{e_{k}(\cdot)=e^{-2 \pi i\left\langle\lambda_{k}, \cdot\right\rangle}: k \in \mathbb{Z}^{2}\right\}$ is an orthonormal basis for $L^{2}\left([0,1]^{2}\right)$, and thus for $L^{2}\left(\left[-\frac{1}{2}, \frac{1}{2}\right]^{2}\right)$. It follows that, on $\left[-\frac{1}{2}, \frac{1}{2}\right]^{2}$,

$$
\hat{f}(\cdot)=\sum_{k \in \mathbb{Z}^{2}}\left\langle\hat{f}, e_{k}\right\rangle e_{k}(\cdot)
$$


in the $L^{2}\left(\left[-\frac{1}{2}, \frac{1}{2}\right]^{2}\right)$ sense, and thus in the $L^{1}\left(\left[-\frac{1}{2}, \frac{1}{2}\right]^{2}\right)$ sense. Note that $\operatorname{supp}(\hat{f}) \subset$ $\left[-\frac{1}{2}, \frac{1}{2}\right]^{2}$. This together with (3.2) leads to

$$
\begin{aligned}
& f(x)=\int_{\mathbb{R}^{2}} d \xi e^{2 \pi i\langle x, \xi\rangle} \hat{f}(\xi) \\
& =\int_{\left[-\frac{1}{2}, \frac{1}{2}\right]^{2}} d \xi e^{2 \pi i\langle x, \xi\rangle} \sum_{k \in \mathbb{Z}^{2}} f\left(\lambda_{k}\right) e_{k}(\xi) \\
& =\sum_{k \in \mathbb{Z}^{2}} f\left(\lambda_{k}\right) \int_{\left[-\frac{1}{2}, \frac{1}{2}\right]^{2}} d \xi e^{2 \pi i\langle x, \xi\rangle} e_{k}(\xi) \\
& =\sum_{k \in \mathbb{Z}^{2}} f\left(\lambda_{k}\right) \frac{\sin \pi\left(x_{1}-k_{1}-\alpha\right) \sin \pi\left(x_{2}-k_{2}-\beta\left(k_{1}\right)\right)}{\pi^{2}\left(x_{1}-k_{1}-\alpha\right)\left(x_{2}-k_{2}-\beta\left(k_{1}\right)\right)}
\end{aligned}
$$

for $x \in \mathbb{R}^{2}$. The proof is completed.

Proof of Theorem 1.3. Necessity. By the same procedure as that of Theorem 1.1, we can show that $\left\{Q+m: m \in \mathbb{Z}^{n}\right\}$ is a tiling, and thus $Q$ is congruent to $[0,1]^{n}$ modulo $\mathbb{Z}^{n}$ by Lemma 2.3 , where $Q=\operatorname{supp}(g)$. Define $\lambda_{j}, S_{j}$ and $Q_{j}$ as

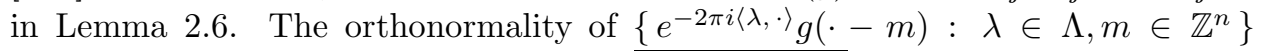
shows $\int_{Q} d x|g(x)|^{2} e^{2 \pi i\langle\lambda, x\rangle}=\int_{\mathbb{R}^{n}} d x g(x) \overline{e^{-2 \pi i\langle\lambda, x\rangle} g(x)}=0$ for $0 \neq \lambda \in \mathbb{Z}^{n}$, and $\int_{Q} d x|g(x)|^{2}=\int_{\mathbb{R}^{n}} d x|g(x)|^{2}=1$. From this, together with Lemma 2.6, it follows that

$$
\begin{aligned}
& \int_{[0,1]^{n}} d x\left(\sum_{j}\left|g\left(x+\lambda_{j}\right)\right|^{2} \chi_{S_{j}}(x)-1\right) e^{2 \pi i\langle\lambda, x\rangle} \\
& =\int_{Q} d x\left(|g(x)|^{2}-1\right) e^{2 \pi i\langle\lambda, x\rangle} \\
& =0
\end{aligned}
$$

for $\lambda \in \mathbb{Z}^{n}$. Applying [16, Theorem 2.4.1] leads to $\sum_{j}\left|g\left(\cdot+\lambda_{j}\right)\right|^{2} \chi_{S_{j}}(\cdot)=1$ a.e. on $[0,1]^{n}$. So $|g(\cdot)|=\chi_{Q}(\cdot)$ by the definition of $Q, S_{j}$ and $Q_{j}$. Define

$$
h(x)= \begin{cases}\frac{g(x)}{|g(x)|}, & \text { if } g(x) \neq 0, \\ 1, & \text { if } g(x)=0 .\end{cases}
$$

Then $g(\cdot)=h(\cdot) \chi_{Q}(\cdot)$, and $h$ is as desired.

Sufficiency. It is obvious that, for $\lambda, \lambda^{\prime}, m, m^{\prime} \in \mathbb{Z}^{n}$,

$$
\begin{aligned}
& I\left(\lambda, m ; \lambda^{\prime}, m^{\prime}\right) \\
& =\int_{\mathbb{R}^{n}} d x e^{-2 \pi i\langle\lambda, x\rangle} g(x-m) \overline{e^{-2 \pi i\left\langle\lambda^{\prime}, x\right\rangle} g\left(x-m^{\prime}\right)} \\
& =\int_{(Q+m) \cap\left(Q+m^{\prime}\right)} d x e^{-2 \pi i\left\langle\lambda-\lambda^{\prime}, x\right\rangle} h(x-m) \overline{h\left(x-m^{\prime}\right)} .
\end{aligned}
$$

If $m \neq m^{\prime}$, then $\left|(Q+m) \cap\left(Q+m^{\prime}\right)\right|=0$ by Lemma 2.3 , and thus $I\left(\lambda, m ; \lambda^{\prime}, m^{\prime}\right)=0$. If $m=m^{\prime}$, then, by Lemma 2.6 ,

$$
I\left(\lambda, m ; \lambda^{\prime}, m^{\prime}\right)=\int_{Q+m} d x e^{-2 \pi i\left\langle\lambda-\lambda^{\prime}, x\right\rangle}=\int_{Q} d x e^{-2 \pi i\left\langle\lambda-\lambda^{\prime}, x\right\rangle}=\delta_{\lambda, \lambda^{\prime}}
$$


So $I\left(\lambda, m ; \lambda^{\prime}, m^{\prime}\right)=\delta_{\lambda, \lambda^{\prime}} \delta_{m, m^{\prime}}$ for $\lambda, \lambda^{\prime}, m, m^{\prime} \in \mathbb{Z}^{n}$. It then follows that $\left\{e^{-2 \pi i\langle\lambda, \cdot\rangle} g(\cdot-m): \lambda \in \Lambda, m \in \mathbb{Z}^{n}\right\}$ is an orthonormal system in $L^{2}\left(\mathbb{R}^{n}\right)$. Now we prove its completeness. Suppose there is an $f \in L^{2}\left(\mathbb{R}^{n}\right)$ such that

$$
\int_{\mathbb{R}^{n}} f(x) e^{2 \pi i\langle\lambda, x\rangle} \overline{g(x-m)}=0
$$

for $\lambda, m \in \mathbb{Z}^{n}$. It is easy to check that this is equivalent to $\int_{Q} f(x+m) \overline{h(x)} e^{2 \pi i\langle\lambda, x\rangle}$ $=0$ for $\lambda, m \in \mathbb{Z}^{n}$. Applying Lemma 2.6, we have $f(\cdot+m) \overline{h(\cdot)}=0$, and thus $f(\cdot+m)=0$ a.e. on $Q$ for $m \in \mathbb{Z}^{n}$. Note that $\bigcup_{m \in \mathbb{Z}^{n}}(Q+m)=\mathbb{R}^{n}$ up to a set of measure 0 by Lemma 2.3. It follows that $f(\cdot)=0$ a.e. on $\mathbb{R}^{n}$, which finishes the proof of the completeness.

\section{ACKNOWLEDGEMENT}

The author would like to thank the referees for their valuable suggestions.

\section{REFERENCES}

[1] Jeffery C. Lagarias, James A. Reeds and Yang Wang, Orthonormal Bases of Exponentials for the $n$-Cube, Duke Math. J., 103(2000), 25-37. MR1758237 (2001h:11104)

[2] Palle E. T. Jorgensen and Steen Pedersen, Spectral Pairs in Cartesian Coordinates, J. Fourier Anal. Appl., 5(1999), 285-302. MR1700084 (2002d:42027)

[3] O. H. Keller, Über die luckenlose Einfüllung des Raumes mit Würfeln, J. Reine und Angew. Math., 163(1930), 231-248.

[4] Jeffery C. Lagarias and P. Shor, Keller's Cube-Tiling Conjecture is False in High Dimensions, Bull. Amer. Math. Soc.(N. S.), 27(1992), 279-283. MR1155280 (93e:52040)

[5] I. Daubechies, Ten Lectures on Wavelets, Philadelphia, 1992. MR.1162107 (93e:42045)

[6] A. Ron and Z. Shen, Weyl-Heisenberg Frames and Riesz Bases in $L^{2}\left(\mathbb{R}^{d}\right)$, Duke Math. J., 89(1997), 237-282. MR1460623 (98i:42013)

[7] Youming Liu and Yang Wang, The Uniformity of Non-uniform Gabor Bases, Adv. Comput. Math., 18(2003), 345-355. MR1968125 (2004h:42036)

[8] O. Christensen, B. Deng and C. Heil, Density of Gabor frames, Appl. Comput. Harm. Anal., 7(1999), 292-304. MF 1721808 (2000j:42043)

[9] Deguang Han and Yang Wang, Lattice Tiling and the Weyl-Heisenberg Frames, Geom. Funct. Anal., 11(2001), 742-758. MR,1866800 (2003j:52021)

[10] M. A. Rieffel, Von Neumann Algebras associated with pairs of lattices in Lie Groups, Math. Ann., 257(1981), 403-413. MF0639575 (84f:22010)

[11] J. Ramanathan and T. Steger, Incompleteness of Sparse Coherent States, Appl. Comput. Harm. Anal., 2(1995), 148-153. MR 1325536 (96b:81049)

[12] J. Benedetto, C. Heil and D. Walnut, Differentiation and the Balian-Low Theorem, J. Fourier Anal. Appl., 1(1995), 355-402. MR1350699 (96f:42002)

[13] Peter G. Casazza and Ole Christensen, Gabor frames over irregular lattices. Frames, Adv. Comput. Math., 18(2003), 329-344. MR1968124 (2004c:42062)

[14] Peter G. Casazza, Ole Christensen and A. J. E. M. Janssen, Weyl-Heisenberg frames, translation invariant systems and the Walnut representation, J. Funct. Anal., 180(2001),85-147. MR $1814424(2002 \mathrm{~b}: 42042)$

[15] J. Wexler and S. Raz, Discrete Gabor expansion, Signal Processing, 21(1990), 207-220.

[16] R. E. Edwards, Fourier series, A Modern Introduction, Volume 1, Second Edition, SpringerVerlag, 1979. MR0545506 (80j:42001)

[17] K. Gröchenig and W. R. Madych, Multiresolution Analysis, Haar Bases, and Self-Similar Tilings of $\mathbb{R}^{n}$, IEEE Transactions on Information Theory, 38(1992), 556-568. MR1162214 (93i:42001)

[18] Alex Iosevich and Steen Pedersen, Spectral and Tiling Properties of the Unit Cube, Internat. Math. Res. Notices, 1998, no. 16, 819-828. MR1643694(2000d:52015) 
[19] X. Dai and D. R. Larson, Wandering Vectors for Unitary Systems and Orthogonal Wavelets, Mem. Amer. Math. Soc., 134(1998), no. 640. MF 1432142 (98m:47067)

[20] Yang Wang, Wavelets, Tiling, and Spectral Sets, Duke Math. J., 114(2002), 43-57. MR.1915035 (2003e:42057)

School of Applied Mathematics and Physics, Beijing University of Technology, BeiJiNG, 100022, PeOple's Republic of China

E-mail address: yzlee@bjut.edu.cn 